\title{
Acute Pancreatitis in Pancreas Divisum Secondary to an Impacted Stone in the Minor Papilla
}

\author{
Rehan Farooqi $^{1}$, Carol Burke ${ }^{2}$, Prabhleen Chahal ${ }^{2}$, Faris El-Khider ${ }^{2}$, Umar Zahid $^{3}$ \\ 1. Internal Medicine, Medstar Union Memorial Hospital, Baltimore, USA 2. Gastroenterology, Cleveland Clinic, \\ Cleveland, USA 3. Internal Medicine, Johns Hopkins Bayview Medical Center, Baltimore, USA
}

Corresponding author: Rehan Farooqi, rehan.m.farooqi@medstar.net

\begin{abstract}
Pancreas divisum is reported to occur in up to $14 \%$ of the population. The majority of patients with this congenital anomaly remain asymptomatic. Pancreas divisum can be associated with recurrent pancreatitis due to inadequate drainage of pancreatic secretions through the dorsal pancreatic duct and the minor papilla. We present a patient with a six-month history of recurrent acute pancreatitis due to an impacted pancreatic duct stone in the minor papilla and an unrecognized pancreas divisum. This situation has only been reported in two other cases in the literature.
\end{abstract}

Categories: Internal Medicine, Medical Education, Gastroenterology

Keywords: pancreas divisum, acute recurrent pancreatitis, minor papilla, pancreatic duct stone, endoscopic ultrasound, cholelithiasis, bile ducts, ercp, major papilla

\section{Introduction}

Pancreas divisum (PD) is a common congenital pancreatic duct anomaly occurring in $4 \%-14 \%$ of the population [1]. It results from a failure of fusion between the dorsal and ventral pancreatic ducts during the seventh week of embryogenesis [1]. Three variants of PD have been described: type 1 is the total failure of fusion of the ventral and dorsal pancreatic ducts; type 2 is the complete absence of the ventral duct; and type 3 , incomplete divisum, is where a small communication is present between the ventral and dorsal pancreatic ducts [2]. In approximately $5 \%$ of patients, the anomaly is associated with recurrent pancreatitis because of the inadequate drainage of pancreatic secretions through the dorsal duct via the minor papilla [2]. In PD, the ventral duct drains the inferior and posterior parts of the head of the pancreas through the major papilla. The dorsal duct drains the superior and anterior parts of the head as well as the body and tail of the pancreas through the minor papilla [3]. We present a patient with recurrent acute biliary pancreatitis in the setting of PD with a dorsal pancreatic duct stone impacted in the minor papilla unrecognized until endoscopic ultrasound (EUS) was performed.

Received 06/17/2019

Review began $07 / 20 / 2019$ Review ended 08/20/2019 Published 08/25/2019

\section{(c) Copyright 2019}

Faroogi et al. This is an open access article distributed under the terms of the Creative Commons Attribution License CC-BY 3.0., which permits unrestricted use, distribution, and reproduction in any medium, provided the original author and source are credited.

\section{Case Presentation}

A 65-year-old male with essential hypertension and a history of heavy alcohol use presented to the hospital with dull, unremitting epigastric pain radiating to the back for the past three weeks. He had associated nausea, early satiety, and anorexia. He had no fever, chills, emesis, jaundice, nor changes in bowel movements. On examination, his blood pressure was $148 / 83 \mathrm{mmHg}$, heart rate was 83 beats per minute, and he was afebrile. No scleral icterus was noted. He had a soft abdomen with mild epigastric tenderness, no palpable organomegaly, and bowel sounds were present. The remainder of his physical examination was noncontributory. Pertinent laboratory tests at that time included a leukocyte count of 4,700 K/uL, hemoglobin $13.2 \mathrm{~g} / \mathrm{dl}$, hematocrit $39 \%$, creatinine $0.72 \mathrm{mg} / \mathrm{dl}$, blood urea nitrogen $4 \mathrm{mg} / \mathrm{dl}$, lipase $250 \mathrm{U} / \mathrm{L}$ (range 16-61 U/L), alkaline phosphatase $168 \mathrm{U} / \mathrm{L}$ (range 32-117 U/L), with normal bilirubin and transaminase levels. Computed tomography (CT) of the abdomen reported a calcified stone in the pancreatic duct with a dilated duct in the body and tail of the pancreas and an additional stone in the duct at the proximal body of the pancreas. An abdominal ultrasound revealed cholelithiasis. The patient underwent endoscopic retrograde cholangiopancreatography (ERCP) with biliary sphincterotomy but pancreatic duct cannulation was unsuccessful. Laparoscopic cholecystectomy was performed for presumed gallstone pancreatitis and the patient was discharged home.

Abdominal pain recurred and five weeks later, the patient was readmitted with acute pancreatitis. Magnetic resonance cholangiopancreatography (MRCP) demonstrated a 5-mm filling defect in the mid pancreatic duct. An ERCP was repeated but attempts at pancreatic duct cannulation were

unsuccessful. Pancreaticoduodenectomy was recommended to the patient, and he was transferred to Cleveland Clinic for a second opinion. Endoscopic ultrasound (EUS) was performed, which demonstrated Type $1 \mathrm{PD}$, an impacted stone at the minor papilla with dorsal duct dilation, and changes of chronic pancreatitis. ERCP was performed next and the minor papilla was found to be bulging (Figure 1). 


\section{Cureus}

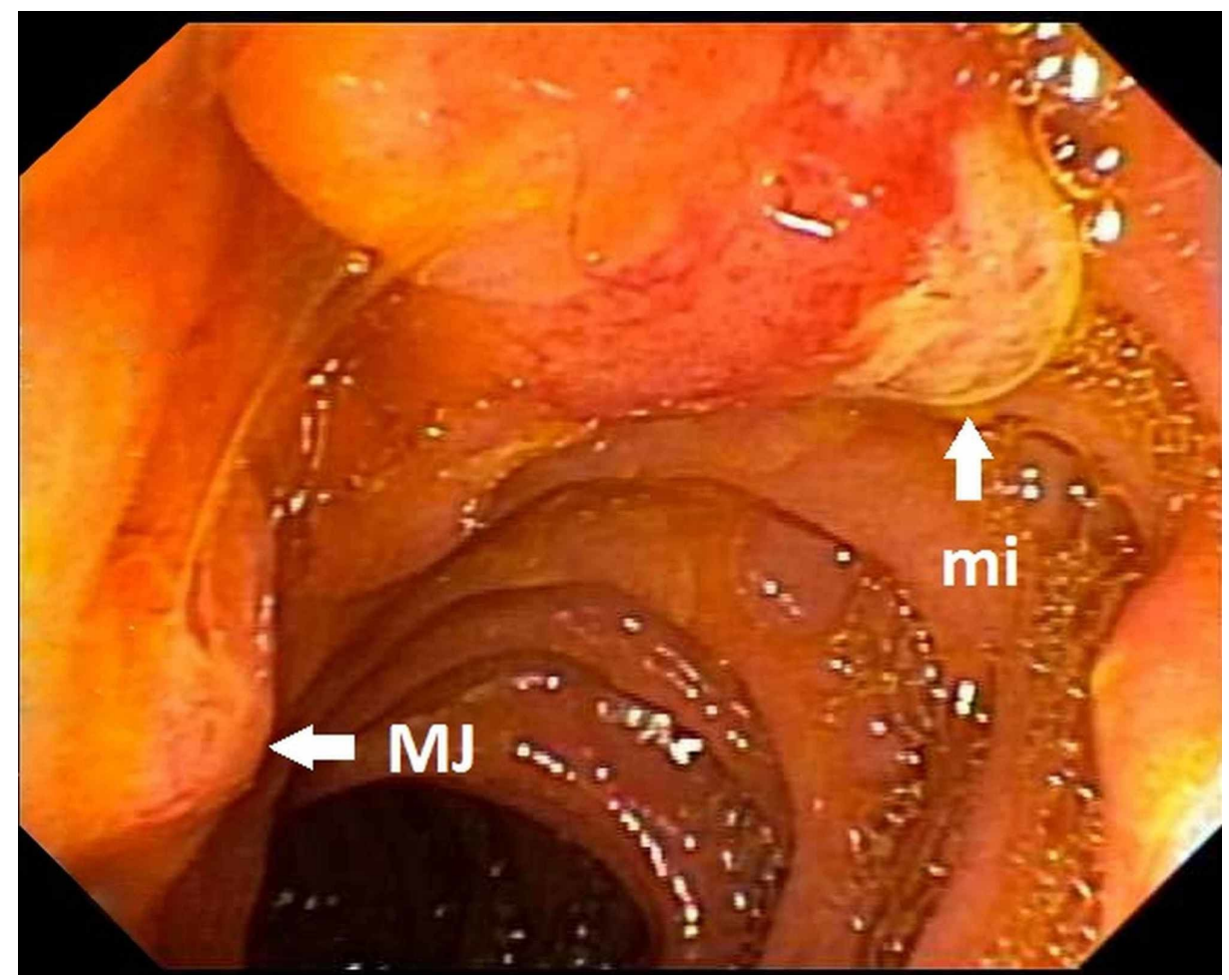

FIGURE 1: Bulging minor papilla (mi) before sphincterotomy and normal-appearing major papilla (MJ)

Dorsal pancreatic sphincterotomy was performed and prompt egress of a single 4-mm stone occurred (Figure 2).

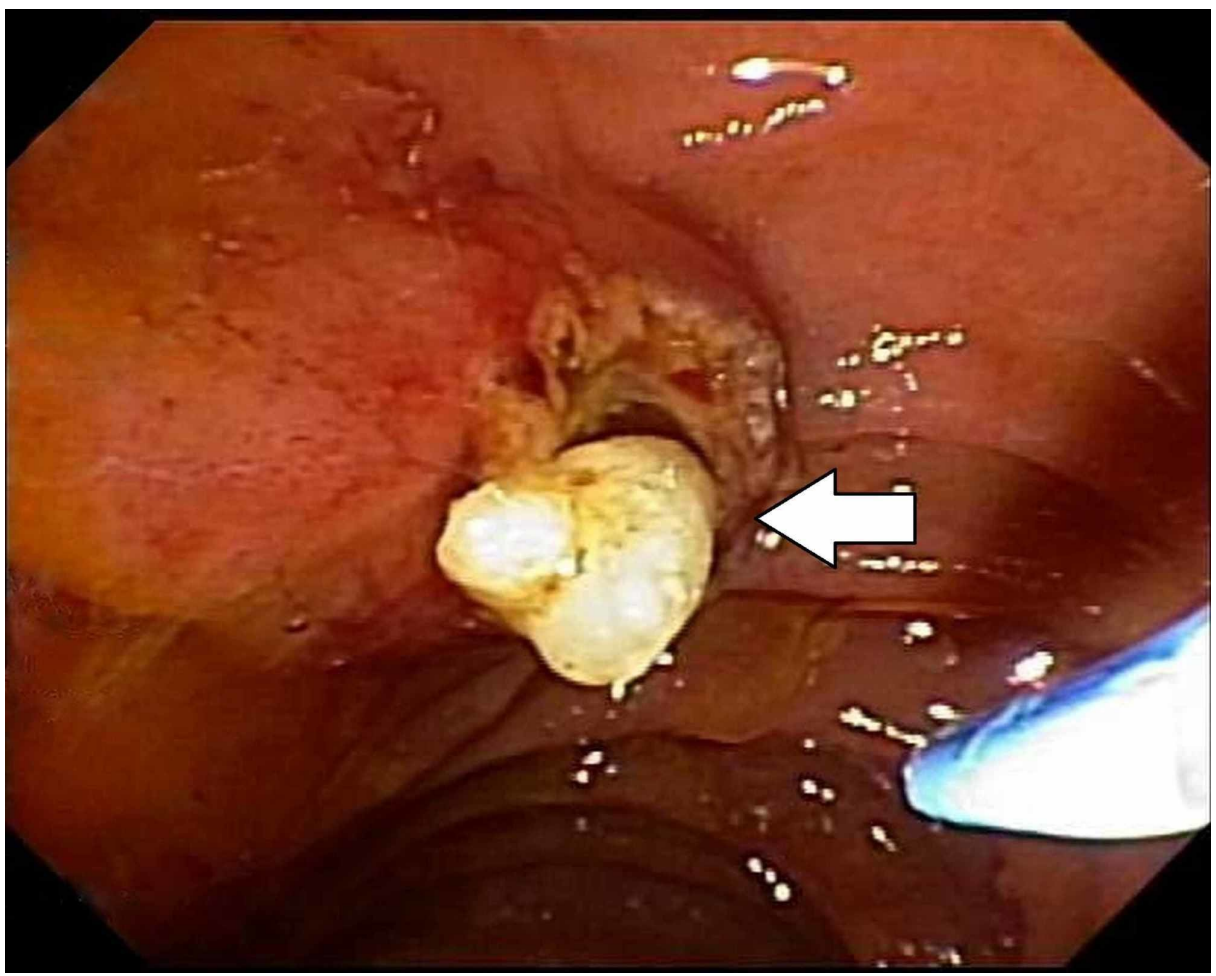

FIGURE 2: The arrow depicts minor papilla stone extraction after sphincterotomy 
A temporary stent was placed into the dorsal pancreatic duct. The patient reported the resolution of his abdominal pain after the procedure. There were no procedure-related complications.

\section{Discussion}

Over $95 \%$ of patients with PD are asymptomatic while the remaining 5\% develop symptoms of acute pancreatitis [4]. In the absence of ductal stones, pancreatitis develops in PD because of increased ductal pressure secondary to insufficient drainage of pancreatic secretions through the minor papilla [4]. Our patient, unfortunately, was in the $5 \%$ category who developed recurrent symptoms of acute pancreatitis. The reason as to why only a select few patients develop symptoms is not clear. The diagnosis of PD is often delayed due to the inadequate sensitivity of conventional radiographic cross-sectional imaging like magnetic resonance imaging (MRI) of the abdomen. The reported accuracy of EUS, MRCP, and multi-detector computed tomography (MDCT) to detect PD is variable and appears to relate to imaging protocols, the expertise of radiologists interpreting the study, and the skill of the endoscopist. In one study, the sensitivity of EUS was $86.7 \%$ higher than the sensitivities of MDCT (15.5\%) and MRCP (60\%) [5]. In a detailed systematic review and meta-analysis, MRCP, secretin-enhanced MRCP, and EUS were compared to address the diagnostic accuracies in the detection of pancreas divisum. It was concluded that EUS was more sensitive at $85 \%$ when compared to MRCP (59\%) and secretin-enhanced MRCP (83\%). All three imaging modalities had specificities above $97 \%$ [6].

This is a unique case with many important clinical points to consider. This is one of three reported cases of acute pancreatitis in a patient with PD due to an impacted stone at the minor papilla [7-9]. The two previously published cases were in 1999 from Switzerland and in 2016 from Japan [8-9]. This challenging case is interesting, as our patient experienced recurrent acute pancreatitis due to an impacted stone in the minor papilla in the setting of PD, which went unrecognized until EUS demonstration of the congenital anomaly. His symptoms resolved after minor papilla pancreatic sphincterotomy with the retrieval of the stone at ERCP. He was symptom-free at the six-month follow-up post-discharge.

\section{Conclusions}

The two most common causes of acute pancreatitis in the United States are alcohol abuse and cholelithiasis. Other causes include trauma, medications, infections, hypertriglyceridemia, pancreas divisum, and hereditary and auto-immune conditions. As internist and specialist, it is important to consider different etiologies of acute pancreatitis and ordering tests cost-effectively to narrow the differential. Our patient quit alcohol several years ago and was status post-cholecystectomy for presumed gallstone pancreatitis prior to presenting to Cleveland Clinic. However, he continued to have recurrent symptoms of acute pancreatitis. The diagnosis of pancreas divisum must be considered for which EUS would be the diagnostic modality of choice, based on its sensitivity and specificity.

\section{Additional Information \\ Disclosures}

Human subjects: Consent was obtained by all participants in this study. Conflicts of interest: In compliance with the ICMJE uniform disclosure form, all authors declare the following: Payment/services info: All authors have declared that no financial support was received from any organization for the submitted work. Financial relationships: All authors have declared that they have no financial relationships at present or within the previous three years with any organizations that might have an interest in the submitted work. Other relationships: All authors have declared that there are no other relationships or activities that could appear to have influenced the submitted work.

\section{References}

1. Schulte S: Embryology, normal variation, and congenital anomalies of the pancreas . Margulis' and Burhenne's Alimentary Tract Radiology. Burhenne HJ (ed): St. Louis: Mosby; 1994. 1039-1051.

2. Turkvatan A, Erden A, Türkoğlu MA, Yener O: Congenital variants and anomalies of the pancreas and pancreatic duct: imaging by magnetic resonance cholangiopancreaticography and multidetector computed tomography. Korean J Radiol. 2013, 14:905-913. 10.3348/kjr.2013.14.6.905

3. Boon N, Delhaye M, Le Moine O, De Maertelaer V, Devière J: Severity of acute gallstone pancreatitis in patients with pancreas divisum. Endoscopy. 2003, 35:407-410. 10.1055/s-2003-38771

4. Izzo P, Di Cello P, Pugliese F, et al.: Pancreas divisum: correlation between anatomical abnormalities and bile precipitation in the gallbladder in seven patients. G Chir. 2016, 37:155-157.

5. Kushnir VM, Wani SB, Fowler K, et al.: Sensitivity of endoscopic ultrasound, multidetector computer tomography and magnetic resonance cholangiopancreatography in the diagnosis of pancreas divisum: a tertiary center experience. Pancreas. 2013, 42:436-441. 10.1097/MPA.0b013e31826c711a

6. Shen Z, Munker S, Zhou B, Li L, Yu C, Li Y: The accuracies of diagnosing pancreas divisum by magnetic resonance cholangiopancreatography and endoscopic ultrasound: a systematic review and metaanalysis. Sci Rep. 2016, 6:35389. 10.1038/srep35389

7. Farooqi R, El-Khider F, Chahal P, Burke CA: Acute pancreatitis in pancreas divisum from an impacted stone in the minor papilla. Am J Gastroenterol. 2018, 113:S766.

8. Isono Y, Matsusaki S, Tanaka $\mathrm{H}$, et al.: An impacted minor papilla stone in a patient with pancreas divisum 


\section{Cureus}

that caused acute exacerbation of chronic pancreatitis: a case report. Nihon Shokakibyo Gakkai Zasshi. 2016, 113:1916-1922. 10.11405/nisshoshi.113.1916

9. Renzulli P, Müller C, Uhl W, Scheurer U, Büchler MW: Impacted papilla minor stone in pancreas divisum causing severe acute pancreatitis: a case for early ERCP in acute pancreatitis of unknown origin. Digestion. 1999, 60:281-283 